DOI: $10.1002 /$ mcda. 301

\title{
Indifference Sets of Reference Points in Multi-Objective Integer Linear Programming
}

\author{
MARIA JOÃO ALVES* and JOÃO CLÍMACO \\ Faculdade de Economia, Universidade de Coimbra / INESC, Coimbra, Portugal
}

\begin{abstract}
Reference point approaches for multi-objective problems rely on the definition of an achievement scalarizing function that projects reference points onto the non-dominated solution set. In this paper, we investigate the behaviour of reference points using a Tchebycheff metric-based scalarizing function in multi-objective pure integer linear programming (MOILP). Since the non-dominated solutions are discrete in MOILP, there are multiple reference points that lead to the same solution, i.e. there are indifference sets on the reference point space. We investigate some properties of the reference points in MOILP and also the graphical representation of indifference sets for tri-objective problems. We further investigate properties of the reference points when additional limitations on the objective function values are introduced. Copyright (C) 2001 John Wiley \& Sons, Ltd.
\end{abstract}

KEY WORDS: integer linear programming; multiple objective analysis; reference points; Tchebycheff metric

\section{INTRODUCTION}

Reference point approaches for dealing with multiobjective programming problems (with integer variables or not) rely on the definition of an achievement scalarizing function - as suggested by Wierzbicki (1980)-by means of reference points that may represent aspiration levels for the objective functions. An achievement scalarizing function projects a reference point onto the nondominated solution set. Tchebycheff metric-based functions have been often used in the definition of achievement scalarizing programs. They are used to compute the non-dominated (efficient) solution closest to a (non-attainable) reference point according to a, possibly weighted, Tchebycheff metric.

Some authors have developed interactive methods using Tchebycheff or other achievement scalarizing functions for dealing with MOILP problems or more general multi-objective problems that also include the integer linear case. Such approaches consider either the variation of

\footnotetext{
*Correspondence to: Faculdade de Economia, Universidade de Coimbra, Av. Dias da Silva, 165, 3004-512 Coimbra, Portugal. E-mail: mjoao@inescc.pt
}

Contract/grant sponsor: PRAXIS XXI; contract/grant number: Project 2/2.1/MAT/465/94 the reference point, occasionally with additional constraints on the objective function values, or the variation of the weights in a (augmented) weighted Tchebycheff metric keeping the reference point constant. Examples of the former type are the approaches from Durso (1992), Vassilev and Narula (1993) Narula and Vassilev (1994) and Karaivanova et al. (1995). The method of Steuer and Choo (1983) is an example of the latter type.

The scalarizing programs used in those interactive approaches are either parameterized on the weights or on the reference point (or even on both) and the variation of the parameters enables to attain different non-dominated solutions. The decision maker (DM) is required to specify the preferences used to assign (implicitly or explicitly) new values to the parameters. But the set of nondominated solutions of an MOILP problem is discrete and there are multiple parameter values that lead to the same solution, i.e. an indifference set on the parameters' space can be defined for each solution. Usually, a method does not provide information about these indifference sets, so the result of a computational phase can be a solution already known. This situation should be avoided, namely in problems where the computational difficulties are considerable such as in integer programming problems. 
In this paper, we examine the indifference sets (regions) on the reference point space. We have centred ourselves on approaches that project reference points onto the non-dominated set through the optimization of a Tchebycheff-based scalarizing program.

This study followed our previous work on the construction of interactive reference point procedures for MOILP problems. We have developed two approaches, which differ only from a technical point of view. The ideas that underlie these approaches were the creation of a simple protocol to interact with the DM-not demanding too much information about his/her preferences - and the reduction of the computational effort, namely by profiting from computations previously performed when producing new non-dominated solutions. In the first approach (Alves and Clímaco, 1999), the scalarizing programs are solved by cutting plane techniques. The combination of cutting planes with the Tchebycheff metric provides a means to perform a postoptimality analysis on the reference point, which is useful for computing new non-dominated solutions. Unfortunately, this method is limited in practice due to the numerical difficulties caused by cutting planes. Thus, we have further investigated a new approach based on branch-and-bound (Alves and Clímaco, 2000) which also addresses multiobjective mixedinteger programs. Both approaches are particularly interesting to perform directional searches for which the DM just chooses an objective function he/she wishes to improve. Using sensitivity analysis, the procedure automatically adjusts the reference point by computing the least variation of one component of the reference point that is needed to 'exit' from the previous non-dominated solution. This automatic tool frees the DM from the task of adjusting the reference point, giving the guarantee that the solution obtained is different from the previous one, and improves the criterion the DM has selected. A technical overview of these approaches is given in Section 2 .

The directional searches provided by our procedures identify particular trajectories of reference points that lead to the same non-dominated solution. But it is clear that many other reference points outside those trajectories may yield the same result. We are referring to the whole (unknown) indifference set on the reference point space corresponding to one nondominated solution, which we also designate as indifference region.
An indifference region on the reference point space is often non-convex, and we have not been able to establish a computationally easy procedure to determine it fully. However, we can easily define a convex indifference sub-region for a nondominated solution using just one reference point that leads to that solution. Furthermore, if we have already known a trajectory of reference points leading to the same solution-a situation that occurs during a directional search-then we can define a larger indifference sub-region. The full (non-convex) indifference region will be the union of such convex sub-regions. In Section 3, we investigate as to how a convex indifference subregion can be determined. We also show with an example how to profit from the directional searches to define larger sub-regions, and briefly report an application that we have been studying.

Independent from using our approaches or other interactive reference point methods, the knowledge of indifference regions, or even subregions, provides the DM with an important insight into the MOILP problem, namely if they can be presented graphically. This information is relevant because

(a) it helps the DM to avoid the selection of reference points that lead to the same solution;

(b) it gives some suggestions on the shape of the non-dominated solution set, such as the number of solutions and their proximity;

(c) it shows a degree of stability of each solution in relation to the change of the reference point.

Moreover, from a theoretical point of view, this study of indifference regions has shown some general properties of the reference points when dealing with MOLIP problems.

The indifference sub-regions we examine herein could also be used within other reference point approaches that either employ directional searches-for instance, methods like Pareto Race (Korhonen and Wallenius, 1988), with a logic developed for MOLP but that could be extended to MOILP-or other approaches that use disjoined reference points-for instance, Durso (1992), Karaivanova et al. (1995) and others. Most of these approaches (including ours) consider additional limitations on the objective function values. The DM interactively specifies bounds for the objective function values (reservation levels), 
which reduce the feasible region of the problem and the non-dominated solutions that are attainable. Reservation levels are often used in interactive multi-objective approaches to guide the search for non-dominated solutions, namely in combination with aspiration levels (usually used as reference points). Thus, a certain reference point may lead to one non-dominated solution or to a different one whether or not additional limitations are imposed on the objective function values. We could still define indifference regions for the nondominated solutions of the restricted problem. In that case, the indifference region of each solution would differ from the corresponding indifference region in the original problem, because the nondominated solution set is only a sub-set of the original one. But, whenever these bounds are temporary, it is more interesting to translate that information into the original problem in order to have a means that provides consistent information during the whole decision process. It is therefore necessary to make a correspondence between the results produced by the use of the same reference points in restricted searches and in unrestricted searches (i.e. in the original problem). This topic is investigated in Section 4.

The study presented in the following sections is oriented to tri-objective problems because the graphical representation of the indifference regions on the reference point space illustrates the addressed issues better.

\section{OVERVIEW OF AN INTERACTIVE DIRECTIONAL SEARCH}

Let us consider the multiple objective integer linear program (MOILP)

$$
\begin{array}{cl}
\max & f_{i}=c^{i} x, i=1, \ldots, k \\
\text { s.t. } & x \in S=\{x \mid A x=b, x \geqslant 0, x \text { integer }\}
\end{array}
$$

where $x$ is an $n$-dimensional vector of variables, $A$ is an $m \times n$ matrix, $b$ is the right-hand side (RHS) vector and the vectors $c^{i}(i=1, \ldots, k)$ have the coefficients of the objective functions (criteria). It is assumed that $S$ is bounded and $c^{i}(i=1, \ldots, k)$ have integer components. Hence, $f_{i}=c^{i} x$ $(i=1 \ldots k)$ are also integer-valued for all feasible $x$.

Let $z^{+}$be a criterion reference point. The scalarizing program $P_{1}\left(z^{+}\right)$projects $z^{+}$onto the (weakly) non-dominated solution set. If $z^{+}$satisfies $z^{+} \geqslant f$ for all the non-dominated points $f$, then the optimal solution of $P_{1}\left(z^{+}\right)$is the (weakly) nondominated solution closest to $z^{+}$according to the Tchebycheff metric. Otherwise, $P_{1}\left(z^{+}\right)$does not minimize a norm but the result is also a (weakly) non-dominated solution. Actually, this minimization does not mean 'coming close' in a traditional sense, but 'coming close or better' (Wierzbicki, 1998). Nevertheless, any $z^{+}$can be replaced by another reference point $z^{++}$which satisfies $z^{++} \geqslant f$, $\forall f$ non-dominated, and produces the same outcome as $z^{+}$(further details about displacing reference points are given in Section 3). So, we call $P_{1}\left(z^{+}\right)$a Tchebycheff scalarizing program.

$$
\begin{array}{ll}
\min & \alpha \\
\text { s.t. } & z_{i}^{+}-c^{i} x \leqslant \alpha, \quad i=1, \ldots, k \\
& x \in S
\end{array}
$$$$
P_{1}\left(z^{+}\right)
$$

In order to avoid weakly non-dominated solutions, which may be obtained when $P_{1}\left(z^{+}\right)$admits alternate optima, an augmented Tchebycheff metric is considered, i.e. ' $m i n{ }^{\prime}$ in $P_{1}\left(z^{+}\right)$is replaced by ' $\min \left\{\alpha-\rho \sum_{i=1}^{k} c^{i} x\right\}$ '. We shall denote this scalarizing program by $P_{2}\left(z^{+}\right)$, where $\rho$ is a small positive constant such that the optimal solution of $P_{2}\left(z^{+}\right)$also optimizes $P_{1}\left(z^{+}\right)$.

The interactive reference point approaches that we have developed (Alves and Clímaco, 1999, 2000) use the scalarizing program $P_{2}\left(z^{+}\right)$to compute non-dominated solutions. At each interaction, the DM can directly assess a new reference point $z^{+}$that is inserted into $P_{2}\left(z^{+}\right)$or just selects an objective function, say $f_{p}$, he/she wants to improve (with respect to the previous non-dominated solution). In the latter case, the reference point is automatically adjusted by increasing the $p$ th component of $z^{+}$, while leaving the other components unchanged, in order to produce new non-dominated solutions throughout a directional search that are more suited to the DM's preferences. This stage involves an iterative process of sensitivity analysis using either cutting plane techniques (Alves and Clímaco, 1999) or branchand-bound techniques (Alves and Clímaco, 2000). The iterative sensitivity analysis procedure determines $\bar{\theta}_{p} \geqslant 0$ such that the reference points between $z^{+}$and $\left(z_{1}^{+}, \ldots, z_{p}^{+}+\bar{\theta}_{p}, \ldots, z_{k}^{+}\right)$lead to the previous non-dominated solution, but a slight increase over $\bar{\theta}_{p}$ leads to a different non-dominated solution (which improves the objective $f_{p}$ ). Only integer reference points are used for MOILP problems because they do not cause loss of 'intermediate' non-dominated solutions (proved in Alves and 
Clímaco, 1999). This means that $P_{2}\left(z^{+}\right)$produces the same non-dominated solution for every $z^{+}=\left(z_{1}^{+}, \ldots, z_{p}^{+}+\theta_{p}, \ldots, z_{k}^{+}\right), \quad 0 \leqslant \theta_{p} \leqslant \bar{\theta}_{p}$, with $\vec{\theta}_{p}$ integer, but $P_{2}\left(\hat{z}^{+}\right)$with $\hat{z}^{+}=\left(z_{1}^{+}, \ldots, z_{p}^{+}+\bar{\theta}_{p}+\right.$ $1, \ldots, z_{k}^{+}$) yields a 'close' but different non-dominated solution.

The cutting plane approach takes advantage of the cutting planes introduced in $P_{2}\left(z^{+}\right)$for solving $P_{2}\left(\hat{z}^{+}\right)$as the cuts remain valid for $P_{2}\left(\hat{z}^{+}\right)$in spite of becoming weaker. In turn, the branch-andbound approach uses the information provided by the branch-and-bound tree that solved $P_{2}\left(z^{+}\right)$. The tree structure and some information on the terminal nodes, which are preserved from one iteration to the next, are used for the sensitivity analysis phase and to obtain the new efficient solution that optimizes $P_{2}\left(\hat{z}^{+}\right)$. The procedure first tries to simplify the tree if new branching is required. This important step, which avoids an ever-growing tree, operates by cutting branches hence discarding parts of the tree. These branches concern variable constraints (such as $x_{i} \leqslant K_{i}$ or $\left.x_{i} \geqslant K_{i}+1\right)$ that were active in the optimum of $P_{2}\left(z^{+}\right)$but are no longer active for $P_{2}\left(\hat{z}^{+}\right)$. This process of profiting from the previous branch-andbound tree in the following computation phase is effective in practice because it reduces the time needed to compute new non-dominated solutions.

\section{INDIFFERENCE REGIONS ON THE REFERENCE POINT SPACE}

It is easy to prove that the optimal solution $x$ of $P_{2}\left(z^{+}\right)$(or $P_{1}\left(z^{+}\right)$) does not change if a constant amount is added to all the components of $z^{+}$. In other words, all the reference points $\left(z_{1}^{+}+\delta, z_{2}^{+}+\right.$ $\delta, \ldots, z_{k}^{+}+\delta$ ) with $\delta \in \mathfrak{R}$ lead to the same nondominated solution-only the $\alpha$ value in $P .\left(z^{+}\right)$ varies. Therefore, every reference point $z^{+} \in \mathfrak{R}^{k}$ can be converted into another one belonging to a hyper-plane, say $Z_{Q}^{+}$, defined by $\sum_{i=1}^{k} z_{i}^{+}=Q$ with $Q \in \Re$ constant. Given $z^{+}=\left(z_{1}^{+}, z_{2-}^{+}, \ldots, z_{k}^{+}\right)$and $Q$, $z^{+}$is converted into $\left(z_{1}^{+}+\bar{\delta}, z_{2}^{+}+\bar{\delta}, \ldots, z_{k}^{+}+\bar{\delta}\right)$ by setting $\bar{\delta}=\left(Q-\sum_{i=1}^{k} z_{i}^{+}\right) / k$. Thus, the reference point space can be represented on a $(k-1)$ dimensional hyper-plane. In particular, for triobjective (bi-objective) problems, the reference point space and its indifference regions (i.e. sets of reference points that lead to the same solution) may be graphically represented in a plane (line). Moreover, there are ranges $\left[z_{i}^{+\min }, z_{i}^{+ \text {max }}\right], i=1, .$. , $k$ such that the variation of each $z_{i}^{+}$within its range allows one to establish the complete nondominated solution set. Therefore, if $Q$ and $z_{i}^{+\min }$, $i=1, . ., k$ are properly defined, the representation of the reference point space may be reduced to

$\bar{Z}_{Q}^{+}=\left\{z^{+} \mid z_{i}^{+} \in\left[z_{i}^{+\min }, z_{i}^{+\max }\right] \forall i, \quad \sum_{i=1}^{k} z_{i}^{+}=Q\right\} \subset Z_{Q}^{+}$ where $z_{i .}^{+\max }=Q-\sum_{j \neq i} z_{j}^{+\min }$. The values of $Q$ and $z_{i}^{+\min }, i=1, \ldots, k$ may be initially set by a heuristic procedure (for instance, based on the values of the pay-off table) keeping the possibility of a further adjustment of $z_{i}^{+m i n}, i=1, \ldots, k$ (by decreasing their values).

Figure 1(a) shows the shape of $\bar{Z}_{Q}^{+}$in the tri-objective case. It is an equilateral triangle whose projection, for example, on plane ' $z_{1}^{+}, z_{2}^{+}$' is the right-angled triangle of Figure 1(b). To simplify the representation, we shall adopt the projection on ' $z_{1}^{+}, z_{2}^{+}$' instead of the equilateral triangle.

Let us turn to the discussion of some properties connected with the behaviour of particular reference points in $P_{2}\left(z^{+}\right)$or $P_{1}\left(z^{+}\right)$.

\section{Proposition 1}

If an efficient solution of the MOILP problem, $x^{a}$, optimizes both $P_{2}\left(z^{+a}\right)$ and $P_{2}\left(z^{+b}\right)$ with $z^{+a}=\left(z_{1}^{+a}, \ldots, z_{p}^{+a}, \ldots, z_{k}^{+a}\right), \quad z^{+b}=\left(z_{1}^{+a}, \ldots, z_{p}^{+a}+\right.$ $\left.\bar{\theta}_{p}, \ldots, z_{k}^{+a}\right), \hat{\theta}_{p}>0$, then $x^{a}$ also optimizes $P_{2}\left(z^{+}\right)$ for $z^{+}$between $z^{+a}$ and $z^{+b}$.

Since Proposition 1 is not difficult to prove, we omit its proof here. This proposition just formalizes a result of the directional searches provided by Alves and Clímaco's approaches: when the DM wants to improve the objective function $f_{p}$, an integer value $\bar{\theta}_{p}$ is automatically determined and added to the $p$ th component of the reference point such that $\theta_{p}$ also leads to the previous efficient solution $\left(x^{a}\right)$, but $\bar{\theta}_{p}+1$ leads to a different efficient solution (say $x^{b}$ ). We recall that only integer reference points are considered because they do not constrain the search for efficient solutions. We can, however, determine a thinner barrier (less than 1) between reference points corresponding to $x^{a}$ and those corresponding to $x^{b}$. It suffices to compare the objective function value of $P_{2}\left(z^{+}\right)$, with $\left(z_{1}^{+a}, \ldots, z_{p}^{+a}+\bar{\theta}_{p}, \ldots\right.$, $\left.z_{k}^{+a}\right) \leqslant z^{+} \leqslant\left(z_{1}^{+a}, \ldots, z_{p}^{+a}+\theta_{p}+1, \ldots, z_{k}^{+a}\right)$, just for $x^{a}$ and $x^{b}$ because one of them optimizes $P_{2}\left(z^{+}\right)$. 


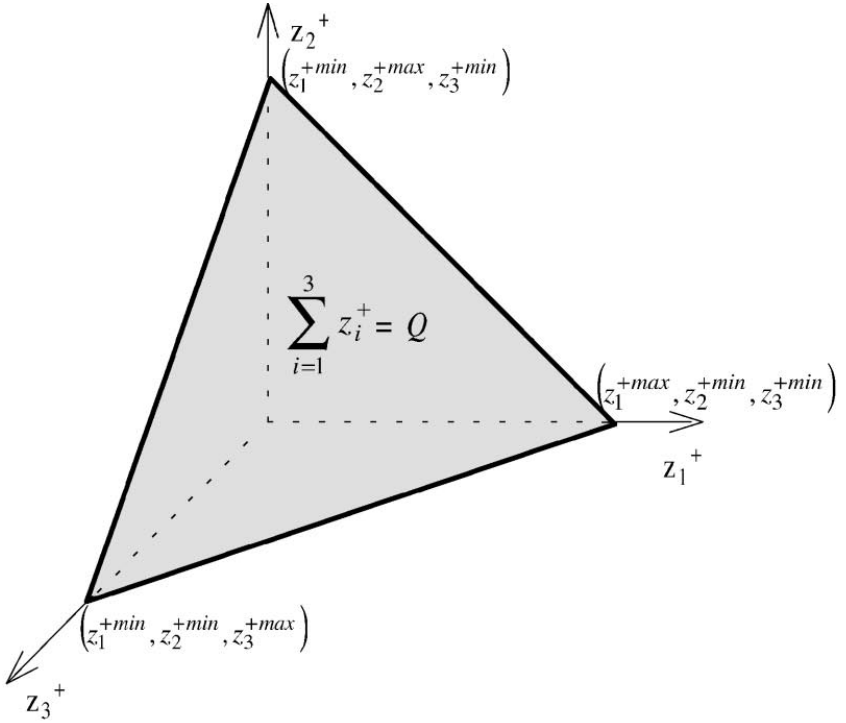

(a)

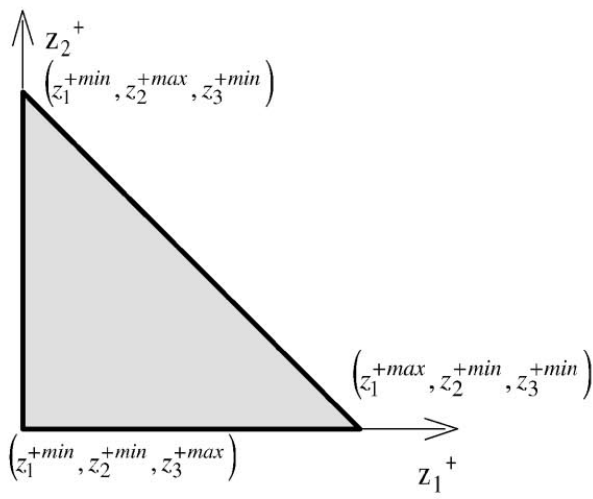

(b)

Figure 1. $\bar{Z}_{Q}^{+}$for tri-objective problems and its projection on ' $z_{1}^{+}, z_{2}^{+}$.

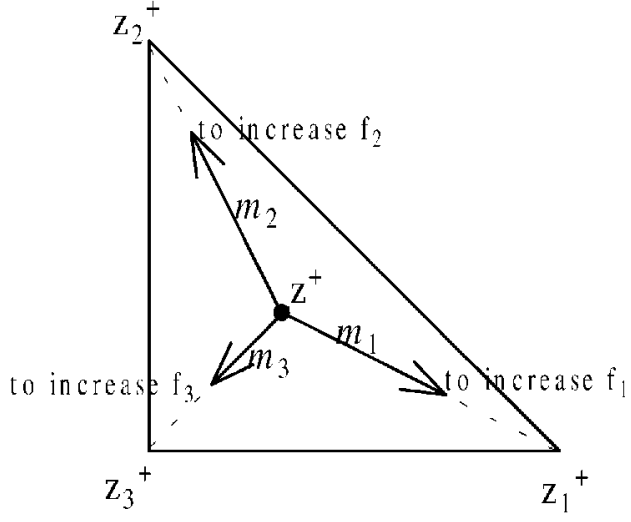

Figure 2. The directions of $z^{+}$used in the directional searches on the projection of $\bar{Z}_{Q}^{+}$onto ' $z_{1}^{+}, z_{2}^{+}$'.

In the tri-objective case, when one component of $\left(z_{1}^{+}, z_{2}^{+}, z_{3}^{+}\right) \in Z_{O}^{+}$is increased by $\theta$, the resulting point can be converted to $Z_{O}^{+}$by subtracting $\frac{\theta}{3}$ from all the components. For instance, $\left(z_{1}^{+}+\theta, z_{2}^{+}, z_{3}^{+}\right)$ is converted into $\left(z_{1}^{+}+\frac{2}{3} \theta, z_{2}^{+}-\frac{1}{3} \theta, z_{3}^{+}-\frac{1}{3} \theta\right)$. Figure 2 shows the three directions $\left(m_{1}, m_{2}\right.$ and $m_{3}$ ) we consider in the directional searches to improve each objective function of tri-objective problems.

Proposition 1 states that if $z^{+a}$ and $z^{+b}$ belong to the same indifference region and a line segment with direction $m_{1}, m_{2}$ or $m_{3}$ can connect them, then all the other points in the line segment also belong to the same indifference region (i.e. there is convexity in these directions).

Let us now denote by $s_{i}, i=1, \ldots, k$, the slack variables associated with the constraints $z_{i}^{+}-c^{i} x \leqslant \alpha, i=1, \ldots, k$, of $P_{2}\left(z^{+}\right)$(or $P_{1}\left(z^{+}\right)$). Thus, $z_{i}^{+}-c^{i} x+s_{i}=\alpha, i=1, \ldots, k$.

\section{Remark 1}

Let $z^{+a}$ be a reference point and $\left(x^{a}, \alpha^{a}, s^{a}\right)$ the optimal solution of $P_{2}\left(z^{+a}\right)$. Let $J \subseteq\{1, \ldots ., k\}$ be the set of active constraints that define the $\alpha^{a}$ value, i.e. $\alpha^{a}=z_{j}^{+a}-c^{j} x^{a}, j \in J$. Therefore, $s_{j}^{a}=0$ for every $j \in J$ and $s_{i}^{a}>0$ for every $i \notin J$. If one or more components $i \notin J$ of $z^{+a}$ are increased by $\delta_{i} \leqslant s_{i}^{a}$, the Tchebycheff distance $\left(\alpha^{a}\right)$ between the reference point and the criterion point $f^{a}$ (image of $\left.x^{a}\right)$ does not change. Thus, this solution remains the closest one to the modified reference point. It follows that all the reference points $\left(z_{1}^{+a}+\delta_{1}, \ldots\right.$, $z_{k}^{+a}+\delta_{k}$ ) with $0 \leqslant \delta_{i} \leqslant s_{i}^{a}, i=1, \ldots ., k$ belong to the indifference region of $x^{a}$.

\section{Example}

Let $z^{+a}=\left(z_{1}^{+a}, z_{2}^{+a}, z_{3}^{+a}\right)$ lead to $x^{a}$ with $s_{1}^{a}>0, s_{2}^{a}>0$ and $s_{3}^{a}=0$. The points $P_{0}=z^{+a}, P_{1}=\left(z_{1}^{+a}+s_{1}^{a}\right.$, $\left.z_{2}^{+a}, z_{3}^{+a}\right), P_{2}=\left(z_{1}^{+a}, z_{2}^{+a}+s_{2}^{a}, z_{3}^{+a}\right), P_{1,2}=\left(z_{1}^{+a}+\right.$ $\left.s_{1}^{a}, z_{2}^{+a}+s_{2}^{a}, z_{3}^{+a}\right)$ and all the points that satisfy 
$\left(z_{1}^{+a}+\delta_{1}, z_{2}^{+a}+\delta_{2}, z_{3}^{+a}\right)$ with $0 \leqslant \delta_{i} \leqslant s_{i}^{a}, i=1,2$, belong to the indifference region of $x^{a}$. A graphical representation of these points converted to $Z_{Q}^{+}$and projected onto ' $z_{1}^{+}, z_{2}^{+}$' is shown in Figure 3.

In tri-objective problems, each indifference region of $Z_{Q}^{+}$projected onto ' $z_{1}^{+}, z_{2}^{+}$, is the union of indifference sub-regions like that of Figure 3. For each $z^{+a}$, there is at least one $s_{j}^{a}=0$ in the optimal solution of $P_{2}\left(z^{+a}\right)$ because $J$ is always non-empty. When just one $s_{j}^{a}$ is zero, the indifference sub-region is a parallelogram, but if two $s_{j}^{a}$ are zero, the parallelogram is reduced to a line segment and if $s_{j}^{a}=0$ for all $j=1, \ldots, 3$, just one point is represented (corresponding to $P_{1,2}$ in Figure 3).

Generally speaking, the reference point $N^{a}=$ $\left(z_{1}^{+a}+s_{1}^{a}, \ldots, z_{k}^{+a}+s_{k}^{a}\right)$-corresponding to $P_{1,2}$ in the previous example-takes a key role in the indifference region of $x^{a}$. It has the following properties: (i) the $\alpha$ optimal value of $P_{2}\left(N^{a}\right)$ is given by the difference between any component of $N^{a}$ and the corresponding component of the criterion point $f^{a}$ (image of $x^{a}$ ); (ii) points $N^{a}$ and $f^{a}$ are converted into the same point on $Z_{O}^{+}$; (iii) any indifference sub-region of reference points that lead to $x^{a}$ (in the sense of Remark 1) includes this reference point. We shall designate such reference points by KERNELS of the indifference regions on the reference point space.

As we have seen, one reference point $z^{+a}$ can be used to state an indifference sub-region on the reference point space corresponding to a nondominated solution $f^{a}$ (cf. Remark 1). If we know several reference points (e.g. through a trajectory)

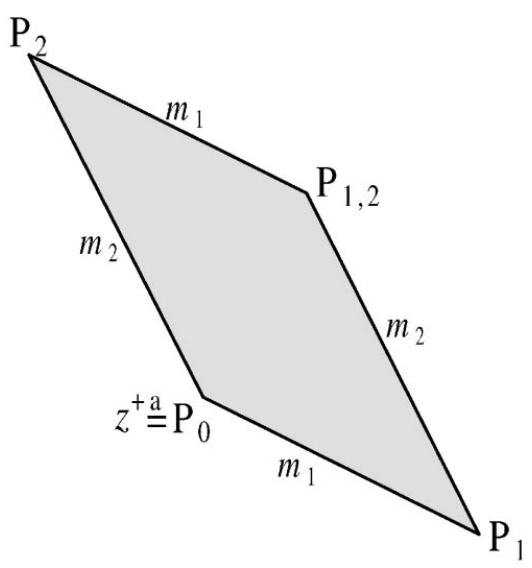

Figure 3. Example of an indifference sub-region. that lead to the same non-dominated solution $f^{a}$, then we will be able to state several indifference sub-regions, whose union defines a larger (sub)region.

So, let us see a way of drawing successive indifference sub-regions for a tri-objective problem profiting from the directional searches described before. Recall that a directional search aims to search for non-dominated solutions that improve one objective function, $f_{1}, f_{2}$ or $f_{3}$, by adjusting the reference point according to the direction $m_{1}, m_{2}$ or $m_{3}$ (Figure 2), respectively. The result is a sequence of discrete non-dominated solutions which successively improve the selected function and a trajectory of (continuous) reference points that lead to each of those solutions. Applying Remark 1 to each reference point on this trajectory, we define successive indifference subregions. As we will see, many of these indifference sub-regions are contained in one of them. Therefore, it is not necessary to take into account all the reference points on the trajectory.

To illustrate these concepts, we start with an example and then analyse the general tri-objective case. The procedure may be used for problems with more than three objective functions although the graphical visualization of the indifference regions is more difficult. In those cases, one possibility could be the representation of cuts of the reference point space by fixing one or more components of the reference points.

We chose a tri-objective multiconstraint knapsack problem with 20 binary variables and 10 constraints and we applied the interactive approach from Alves and Clímaco (2000) to this problem. We started by choosing the reference point $(231,275,262)$, and a first non-dominated solution, $f^{H}=(117,162,170)$, was computed. Then we chose the first objective function $\left(f_{1}\right)$ to be improved in relation to $f^{H}$. The procedure automatically updated the reference point to $(271,275,262)$ which yielded a new nondominated solution, $f^{A}=(173,160,109)$. The reference point $(271,275,262)$ is the first integer point in the trajectory $(117+\theta, 275,262), \theta>0$ that is able to get away from $f^{H}$. Consequently, $(270,275,262)$ is the largest integer point in this trajectory that leads to $f^{H}$. On continuing the search through the same direction, the nondominated solution $f^{B}=(199,185,81)$ was computed by considering the reference point $(354,275,262)$. The following diagram gives a brief summary of this search. 
Efficiont sulution:

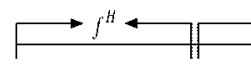

Reference point: $(231.275 .262)(270, ", " 1(271 . ")$.

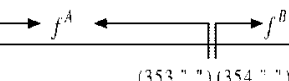

We omit here the technical details of the way the sensitivity analysis updates the reference point since they are not important for computing indifference sub-regions on the reference point space. The relevant information is only that the reference points $(231+\theta, 275,262), 0 \leqslant \theta$ $\leqslant 39$ lead to solution $f^{H}$ and the reference points $(271+\theta, 275,262), 0 \leqslant \theta \leqslant 82$ lead to solution $f^{A}$. These reference points can be used to define indifference sub-regions for $f^{H}$ and $f^{A}$, respectively. Note that other directional search approaches could also be used to give this kind of information.

Let us consider just the solution $f^{A}$ for illustrating the computation of indifference subregions. According to Remark 1, each reference point from $(271,275,262)$ to $(353,275,262)$ can define a sub-region for $f^{A}$. The first $k(k=3)$ constraints of $P_{2}\left(z^{+}\right)$, which are $z_{i}^{+}-c^{i} x \leqslant \alpha$ $(i=1, \ldots, 3)$, are assigned the following values: $z^{+}$is the parametric reference point $(271+\theta, 275$, 262), $\quad 0 \leqslant \theta \leqslant 82, \quad$ and $\quad\left(c^{1} x, c^{2} x, c^{3} x\right)=f^{A}=$ $(173,160,109)$. Hence, in the optimal solution of $P_{2}\left(z^{+}\right)$the constraints become $98+\theta \leqslant \alpha, 115 \leqslant \alpha$, $153 \leqslant \alpha$, respectively for $i=1,2,3$, with $\alpha$ the minimum value that satisfies these constraints. The set of active constraints $J \subseteq\{1, \ldots, 3\}$ varies within the range $0 \leqslant \theta \leqslant 82$, occurring in the following situations: for $0 \leqslant \theta<55, \quad J=\{3\}$ and the $\alpha$ optimal value is set by the third constraint $(\alpha=153)$, but for $55<\theta \leqslant 82, J=\{1\}$ and the $\alpha$ optimal value is set by the first constraint $(\alpha=98+\theta)$; for $\theta=55, J=\{1,3\}$. To sum up, the slack variables vary qualitatively in the following way:

$0 \leqslant \theta<55 \Rightarrow s_{1}>0, s_{2}>0, s_{3}=0$

$\theta=55 \Rightarrow s_{1}=0, s_{2}>0, s_{3}=0$

$55<\theta \leqslant 82 \Rightarrow s_{1}=0, s_{2}>0, s_{3}>0$

For $0 \leqslant \theta<55$ and $55<\theta \leqslant 82$, each reference point enables to define a parallelogram as an indifference sub-region; for $\theta=55$, the parallelogram is reduced to a line segment. Let us first analyse the parallelograms defined for $0 \leqslant \theta \leqslant 55$.
First part: $0 \leqslant \theta \leqslant 55$

Let us consider the indifference sub-regions of $f^{A}$ defined by the reference points $(271+\theta, 275$, 262) with $0 \leqslant \theta \leqslant 55$. According to Remark 1 , all the points $\left(271+\theta+\delta_{1}, 275+\delta_{2}, 262+\delta_{3}\right)$, $0 \leqslant \theta \leqslant 55, \quad 0 \leqslant \delta_{i} \leqslant s_{i} \quad(i=1, \ldots, 3)$ belong to the indifference region of $f^{A}$. The values of the slack variables $s_{i}$ are set by the first constraints of $P_{2}\left(z^{+}\right)$, i.e. $98+\theta+s_{1}=\alpha, \quad 115+s_{2}=\alpha$ and $153+s_{3}=\alpha$. The optimal value of $\alpha$ is 153 for $0 \leqslant \theta \leqslant 55$, thus $s_{1}=55-\theta, s_{2}=38$ and $s_{3}=0$. Hence, for a particular $\theta=\theta^{\prime}$, the corner points that define the indifference sub-region are:

$P_{0}^{\theta^{\prime}}:$ the original

$$
\left(271+\theta^{\prime}, 275,262\right)
$$

$P_{1}^{\theta^{\prime}}:$ adding $s_{1}$

$P_{2}^{\theta^{\prime}}$ : adding $s_{2}$

$\left(271+\theta^{\prime}, 275+38,262\right)$

$P_{1,2}^{\theta^{\prime}}:$ adding $s_{1}$ and $s_{2}$

$(271+55,275+38,262)$

As stated before, a reference point $z^{+}$can be converted to a plane $Z_{Q}^{+}$by adding $\left(Q-\sum_{i=1}^{k} z_{i}^{+}\right) / k$ to all the components of $z^{+}$. This conversion enables the representation in a plane of the indifference sub-regions (parallelograms). Considering $Q=500$, the above points are converted into the following ones:

$$
\begin{aligned}
& P_{0}^{\theta^{\prime}}:\left(168.33+\frac{2}{3} \theta^{\prime}, 172.33-\frac{1}{3} \theta^{\prime}, 159.33+\frac{1}{3} \theta^{\prime}\right) \\
& P_{1}^{\theta^{\prime}}:(205,154,141) \\
& P_{2}^{\theta^{\prime}}:\left(155.67+\frac{2}{3} \theta^{\prime}, 197.67-\frac{1}{3} \theta^{\prime}, 146.67-\frac{1}{3} \theta^{\prime}\right) \\
& P_{1,2}^{\theta^{\prime}}:(192.33,179.33,128.33)
\end{aligned}
$$

Now the sum of the components of each point is equal to $Q=500$. The point $P_{1,2}^{\theta^{\prime}}$ is independent of $\theta^{\prime}$ because it is the KERNEL $\left(N^{A}\right)$ of the indifference region of $f^{A}$. Figure 4 shows a rough draft of the superposed parallelograms defined by the two extreme parameter values, $\theta=0$ and 55 (just a line segment), and two intermediate values, $\theta=10$ and 20 . From $\theta=$ 0 to 55 the parallelograms become successively smaller, so that the parallelogram for $\theta=0$ covers the others. Therefore, it would be enough to consider just the indifference sub-region defined by the first reference point, i.e. $(271,275$, 262).

Let us now analyse the parallelograms defined by the reference points $(271+\theta, 275,262)$ with $55 \leqslant \theta \leqslant 82$. 


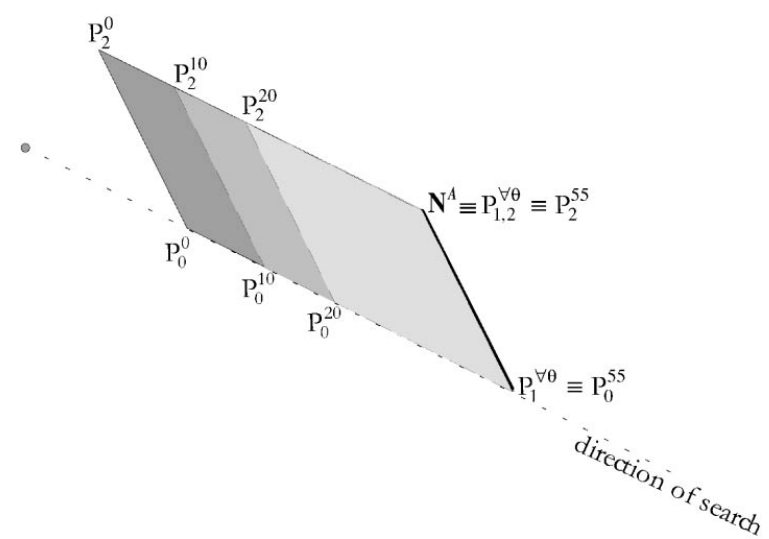

Figure 4. Draft of superposed indifference sub-regions of $f^{A}$ (1st part).

Second part: $55 \leqslant \theta \leqslant 82, \quad s_{1}=0, \quad s_{2}=\theta-17$, $s_{3}=\theta-55$.

Let us consider the variable's change $\phi=\theta-55$. Hence, $0 \leqslant \phi \leqslant 27, s_{1}=0, s_{2}=\phi+38, s_{3}=\phi$. For a particular $\phi=\phi^{\prime}$, the corner points that define the indifference sub-region are:

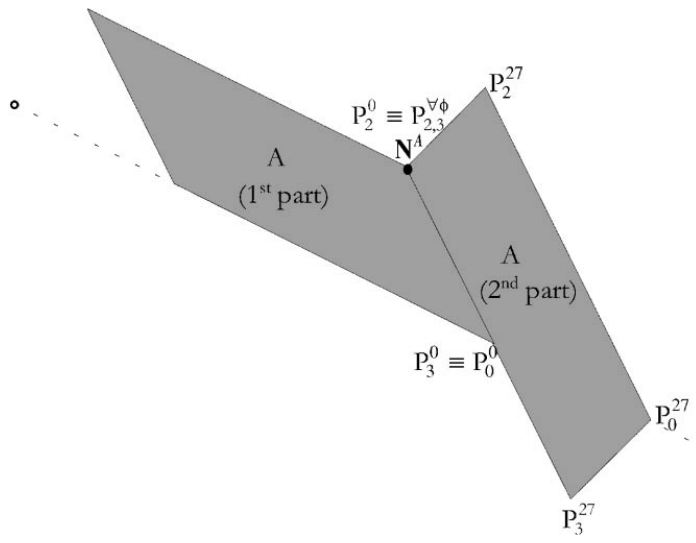

Figure 5. Draft of indifference sub-regions of $f^{A}$ (1st and 2nd parts).

indifference region is defined. But if more than one variable $s_{i}$ is zero, the parallelogram is reduced to a line segment or a point, respectively, for two or three $s_{i}$ variables equal to zero. Let us consider that the region is a parallelogram since a line segment or a point are particular cases of the

$P_{0}^{\phi^{\prime}}:$ the original

$$
\left(326+\phi^{\prime}, 275,262\right) \quad \frac{\text { conversion }}{Z_{\mathrm{Q}}^{+}(Q=500)}
$$

$\left(205+\frac{2}{3} \phi^{\prime}, 154-\frac{1}{3} \phi^{\prime}, 141-\frac{1}{3} \phi^{\prime}\right)$

$P_{2}^{\phi^{\prime}}:$ adding $s_{2}$

$\left(326+\phi^{\prime}, 275+38+\phi^{\prime}, 262\right)$

$\left(192.33+\frac{1}{3} \phi^{\prime}, 179.33+\frac{1}{3} \phi^{\prime}, 128.33-\frac{2}{3} \phi^{\prime}\right)$

$P_{3}^{\phi^{\prime}}:$ adding $s_{3}$

$\left(326+\phi^{\prime}, 275,262+\phi^{\prime}\right)$

$\left(205+\frac{1}{3} \phi^{\prime}, 154-\frac{2}{3} \phi^{\prime}, 141+\frac{1}{3} \phi^{\prime}\right)$

$P_{2,3}^{\phi^{\prime}}$ : adding $s_{2}$ and $s_{3}\left(326+\phi^{\prime}, 275+38+\phi^{\prime}, 262+\phi^{\prime}\right)$

$(192.33,179.33,128.33)$

Now the parallelograms become larger from $\phi=0$ to 27 , thus they are all covered by the last one. The indifference sub-region defined by the last reference point, $(353,275,262)$ corresponding to $\phi=27$, is shown in Figure 5. It is appended to the larger indifference sub-region defined in the first part of this analysis.

To sum up, we can state a systematic way to define indifference sub-regions and to represent them graphically for the tri-objective case.

(1) Let us suppose that we know one reference point $z^{+}$that leads to the non-dominated solution $f^{a}$ through the optimization of $P_{2}\left(z^{+}\right)$. If only one variable $s_{i}(i=1, \ldots, 3)$ is zero in the optimal solution of $P_{2}\left(z^{+}\right)$, then a parallelogram-shaped latter, and assume that the slack variables $s_{i}$ $(i=1, \ldots, 3)$ satisfy $s_{p}>0, s_{q}>0$ and $s_{r}=0$, $p, q, r \in\{1,2,3\}, p \neq q \neq r$ in the optimal solution of $P_{2}\left(z^{+}\right)$. To represent the parallelogram graphically, first it is necessary to convert $z^{+}$into a point $z^{++}$that belongs to a plane $Z_{Q}^{+}$(as stated before) and define the KERNEL $N^{a} \in Z_{Q}^{+}$. $N^{a}$ is the only point belonging to the indifference region of $f^{a}$ for which $s_{i}=0, i=1, \ldots, 3$, and it corresponds to the conversion of $f^{a}$ to $Z_{Q}^{+}$. The parallelogram can be drawn directly in the following way: draw $z^{++}$ and the KERNEL $N^{a}$; draw two lines from $z^{++}$, one in the direction $m_{p}$ and the other in the direction $m_{q}$; then, draw two lines from $N^{a}$ that are parallel to each one of the lines drawn from $z^{++}$. The points $z^{++}, N^{a}$ and the line intersection 
points define the parallelogram, which is an indifference sub-region of $f^{a}$.

(2) Let us now suppose that we know a range $\left[z^{+a}, z^{+b}\right]$ of reference points, which differ in one component (i.e. belong to one of the directions $m_{1}$, $m_{2}$ or $m_{3}$ ), that lead to the non-dominated solution $f^{a}$. If the set $\mathbf{J}$ of active constraints changes within $\left[z^{+a}, z^{+b}\right]$, then the range $\left[z^{+a}, z^{+b}\right]$ must be split into sub-ranges. In that case, there is a splitting point $z^{+c}$ that divides $\left[z^{+a}, z^{+b}\right]$ into $\left[z^{+a}\right.$, $\left.z^{+c}\right]$ and $\left[z^{+c}, z^{+b}\right]$, for which $J^{c}=J^{a} \cup J^{b}$. The number of variables $s_{i}(i=1, \ldots, 3)$ equal to zero in $P_{2}\left(z^{+c}\right)$ (i.e. the cardinality of $J^{c}$ ) is one more than in $P_{2}\left(z^{+}\right)$with $z^{+} \in\left[z^{+a}, z^{+c}[\cup] z^{+c}, z^{+b}\right]$. Each reference point in $\left[z^{+a}, z^{+b}\right]$ allows drawing an indifference sub-region of $f^{a}$ but, for each sub-range there always exists one reference point whose indifference sub-region covers the others: $z^{+a}$ 'covers' $\left[z^{+a}, z^{+c}\right], z^{+b}$ 'covers' $\left[z^{+c}, z^{+b}\right]$, and one of the extreme points $\left(z^{+a}\right.$ or $z^{+b}$ ) 'covers' $\left[z^{+a}, z^{+b}\right]$ if there is no need to split the range. Therefore, it is enough to follow the steps in point (1) with $z^{+a}, z^{+b}$ or both to draw the larger indifference sub-region of $f^{a}$ defined by $\left[z^{+a}, z^{+b}\right]$.

We recall that this procedure does not define the whole indifference region of one solution but only some of its convex sub-regions. This procedure may be integrated into an interactive method, which implies that sub-regions are interactively appended and presented to the DM. The intention is not to compute all the indifference regions on the reference point space but to help the DM in the decision process, namely in the selection of new reference points. However, we have computed all the indifference regions of the previous problem in order to give a better illustration of the shape of these regions. They are shown in Figure 6 in the triangle $\bar{Z}_{Q}^{+}$defined by $Q=500$ and $z_{i}^{+\min }=50(i=1, \ldots, 3)$. Note that the indifference regions are, in general, nonconvex but the lines that delimit them have particular slopes $\left(m_{1}, m_{2}\right.$ or $\left.m_{3}\right)$. This is true for any tri-objective MOILP problem, and the extension for more than tri-objective problems is straightforward.

The sub-regions corresponding to solutions $f^{H}$ and $f^{A}$ determined during the directional search by the procedure described above are delimited by a grey borderline in Figure 6. All the KERNELS are also marked in Figure 6. Table I presents the values of the corresponding non-dominated solutions.
Table I. All the non-dominated solutions of the example

\begin{tabular}{lrrr}
\hline & $f_{1}$ & \multicolumn{1}{c}{$f_{2}$} & \multicolumn{1}{c}{$f_{3}$} \\
\hline A & 173 & 160 & 109 \\
B & 199 & 185 & 81 \\
C & 48 & 109 & 174 \\
D & 39 & 206 & 97 \\
E & 0 & 89 & 221 \\
F & 39 & 188 & 157 \\
G & 28 & 127 & 182 \\
H & 117 & 162 & 170 \\
I & 72 & 95 & 188 \\
J & 84 & 107 & 181 \\
K & 117 & 187 & 136 \\
L & 100 & 192 & 110 \\
M & 0 & 96 & 198 \\
\hline
\end{tabular}

The indifference regions on the reference point space have already shown their potentialities in an application under study. The application concerns the selection of remote load control strategies in an electric distribution network. The problem, which involves different conflicting aspects such as reducing peak demand, maximizing utility profits and minimizing discomfort to consumers, has been modelled as a tri-objective MOILP problem. It was proposed and studied using the STEM method by Jorge et al. (2000). The STEM method (Benayoun et al., 1971) is an interactive method in which the DM must specify, at each interaction, relaxation quantities for the objectives he/she considers satisfactory in order to improve the remaining ones. In Jorge et al (2000), five nondominated solutions were computed using the STEM method. However, besides the difficulties of choosing the relaxation quantities, the STEM does not give any suggestion on whether the problem has a large number of non-dominated solutions or not, or on the magnitude of the differences among 'intermediate' solutions. Instead, the use of directional searches, attached to the display of indifference (sub)regions, presents other information on this problem. For instance, successive solutions obtained through a directional search sometimes have very close criterion values but there are also 'abrupt jumps' that lead to very different criterion values. We may further expect that the non-dominated set contains a large number of solutions, as suggested by the filled areas in the triangle of Figure 7 for the 30 nondominated solutions already computed. The indifference (sub)regions presented in this graph also 


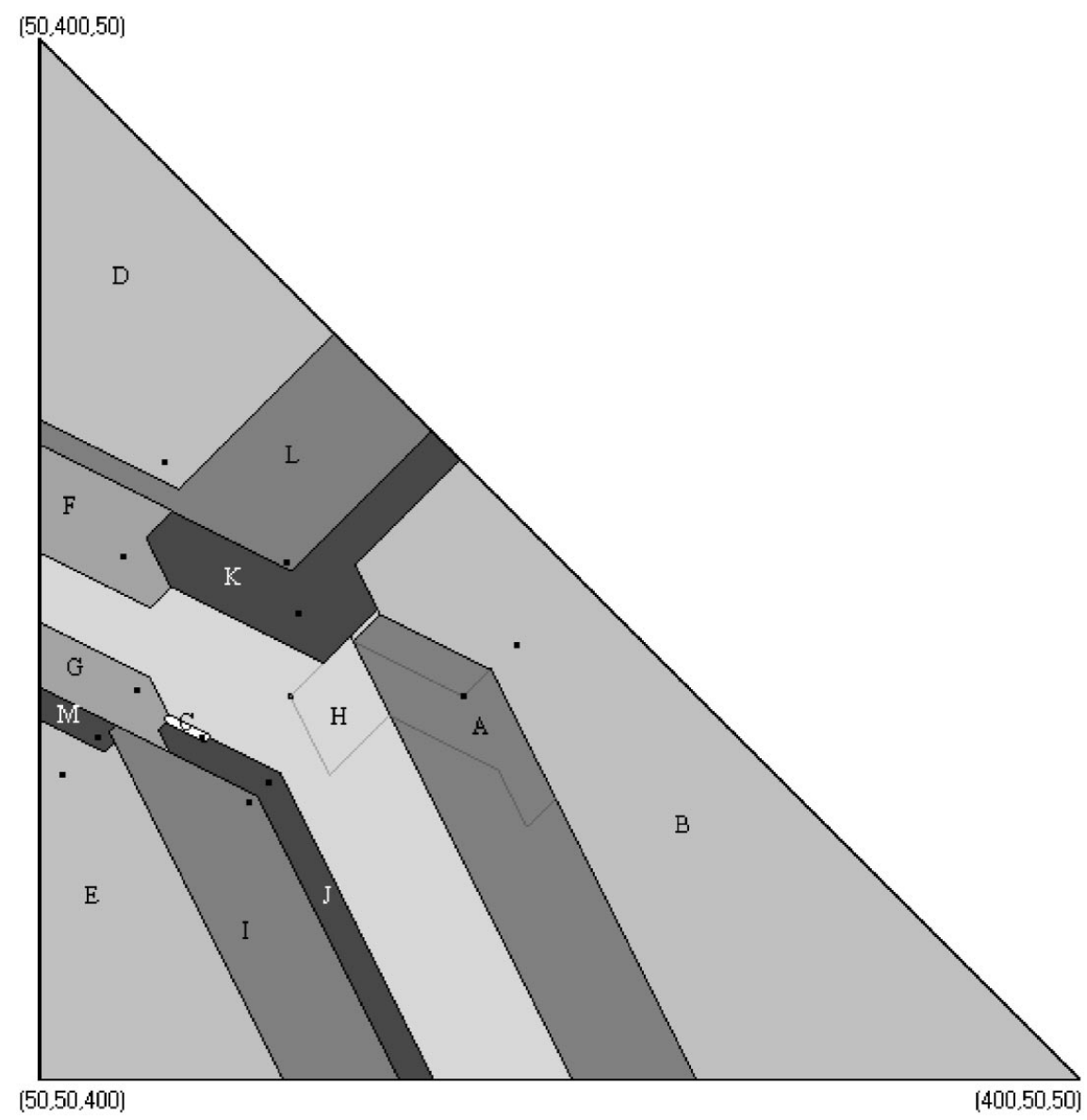

Figure 6. All the indifference regions of the example.

give an idea of the stability degrees of the nondominated solutions with respect to the intention of improving one criterion.

\section{CONSIDERING ADDITIONAL LIMITATIONS ON THE OBJECTIVE FUNCTION VALUES}

Throughout a search process for non-dominated solutions, the DM may wish to impose additional limitations (bounds) on the objective function values. In reference point approaches, like those we have been considering, these additional constraints are included in the scalarizing program $P_{2}\left(z^{+}\right)$or $P_{1}\left(z^{+}\right)$. In this section we further investigate the representation, on the original reference point space, of indifference regions corresponding to non-dominated solutions that have been computed by scalarizing programs with additional limitations on the criteria.

For simplification, let us consider that additional limitations $c^{j} x \geqslant L_{j}, j \in \bar{K} \subseteq\{1, \ldots, k\}$ are introduced in $P_{1}\left(z^{+}\right)$(instead of $P_{2}\left(z^{+}\right)$). Let this scalarizing program be $P_{1}\left(z^{+}, L\right)$ :

$$
\begin{array}{ll}
\min & \alpha \\
\text { s.t. } & z_{i}^{+}-c^{i} x \leqslant \alpha, \quad P_{1}\left(z^{+}, L\right) \\
& c^{j} x \geqslant L_{j} \quad \forall j \in \bar{K} \\
& x \in S
\end{array}
$$

The introduction of $c^{j} x \geqslant L_{j}, \quad j \in \bar{K}$ may restrict the non-dominated solution set that is attainable. This means that, for a given reference 

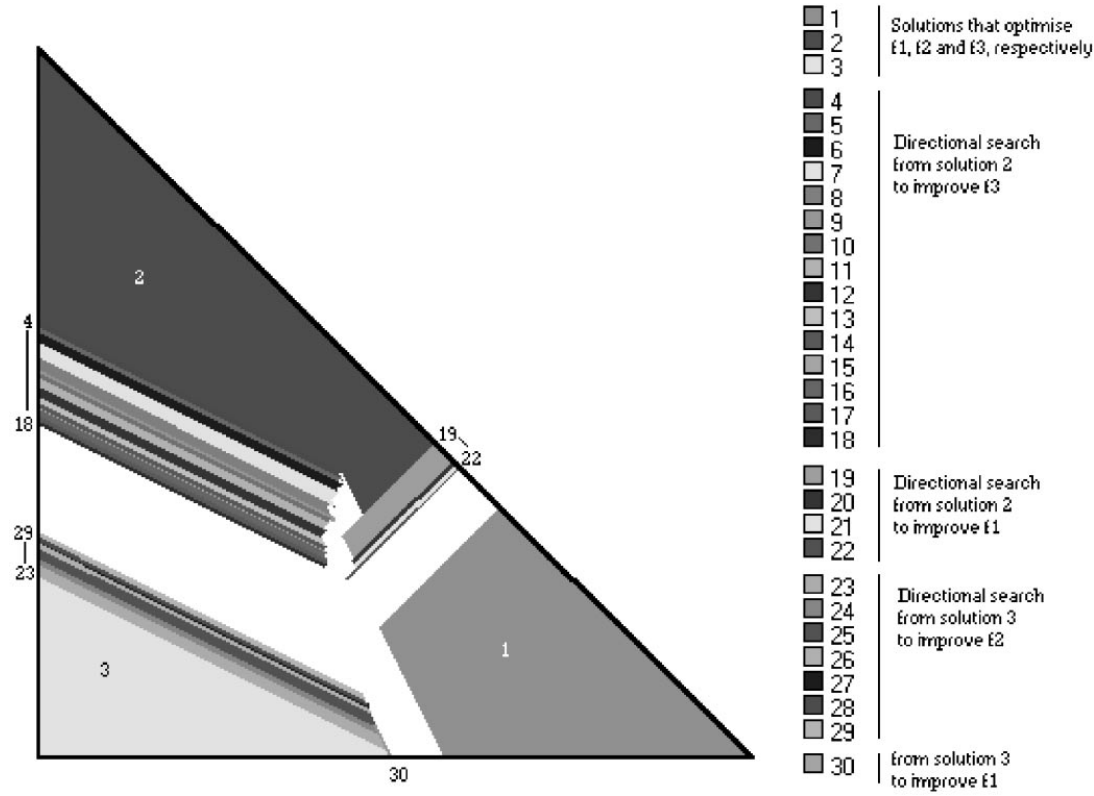

Figure 7. Some indifference sub-regions of a problem for selection of remote load control strategies.

point, a non-dominated solution that optimizes $P_{1}\left(z^{+}, L\right)$ may be non-optimal for $P_{1}\left(z^{+}\right)$. Thus, only some non-dominated solutions have representation on the reference point space of the restricted multi-objective problem and the decomposition of this space is different from the decomposition of the original problem. In order to have a consistent graph that can present any non-dominated solution of the original problem, regardless of how it has been computed, it is necessary first to map reference points $z^{+}$onto $z^{++}$ such that $P_{1}\left(z^{+}, L\right)$ and $P_{1}\left(z^{++}\right)$have the same outcome.

The following lemma establishes a situation for which the results of $P_{1}\left(z^{+}, L\right)$ and $P_{1}\left(z^{+}\right)$are equal.

\section{Lemma 1}

Let $(\tilde{x}, \tilde{\alpha})$ be the optimal solution of $P_{1}\left(z^{+}, L\right)$ for a given $z^{+}$. If $z_{j}^{+} \geqslant L_{j}+\tilde{\alpha}$ for all $j \in \bar{K}$, then $(\tilde{x}, \tilde{\alpha})$ also optimizes $P_{1}\left(z^{+}\right)$. (This means that the limitations $c^{j} x \geqslant L_{j}, \forall j \in \bar{K}$ could be dropped because they do not constrain the result).

\section{Proof}

Suppose that at least one limitation $c^{r} x \geqslant L_{r}$, $r \in K$ constrains the result of the scalarizing program. This means that, although $(\tilde{x}, \tilde{\alpha})$ is a feasible solution of $P_{1}\left(z^{+}\right)$, it is not an optimal solution of $P_{1}\left(z^{+}\right)$. Let $(\hat{x}, \hat{\alpha})$ be an optimal solution of $P_{1}\left(z^{+}\right)$and $c^{r} \hat{x}<L_{r}$ obviously holds.

The constraint $c^{r} x+\alpha \geqslant z_{r}^{+}$in $P_{1}\left(z^{+}\right)$imposes that $c^{r} \hat{x}+\hat{\alpha} \geqslant z_{r}^{+}$. Since $z_{r}^{+} \geqslant L_{r}+\tilde{\alpha}$, then $c^{r} \hat{x}+\hat{\alpha} \geqslant z_{r}^{+} \geqslant L_{r}+\tilde{\alpha} \Rightarrow c^{r} \hat{x} \geqslant L_{r}+\tilde{\alpha}-\hat{\alpha} . \quad$ But $c^{r} \hat{x}<L_{r}$, so, $L_{r}+\tilde{\alpha}-\hat{\alpha} \leqslant c^{r} \hat{x}<L_{r}$ implying that $\tilde{\alpha}<\hat{\alpha}$, which contradicts the hypothesis that $(\hat{x}, \hat{\alpha})$ optimizes $P_{1}\left(z^{+}\right)$.

Proposition 2 states a systematic way of obtaining a reference point that leads to the same solution as the one produced by another reference point when projected onto the non-dominated solution set restricted by criterion bounds. The reference points will be equal if the criterion bounds do not constrain the outcome of $P_{1}\left(z^{+}, L\right)-$ Lemma $1-$ but they will be different otherwise.

\section{Proposition 2}

If $(\tilde{x}, \tilde{\alpha})$ optimizes $P_{1}\left(z^{+}, L\right)$, then it also optimizes $P_{1}\left(z^{++}\right)$with $z_{i}^{++}=z_{i}^{+}$for $i \notin \bar{K}$ and $z_{j}^{++}=$ $\max \left\{z_{j}^{+}, L_{j}+\tilde{\alpha}\right\}$ for $j \in \bar{K}$.

\section{Proof}

(1) Since $c^{j} \tilde{x} \geqslant L_{j}, \Leftrightarrow c^{j} \tilde{x}+\tilde{\alpha} \geqslant L_{j}+\tilde{\alpha}, \forall j \in \bar{K}$, and $c^{j} \tilde{x}+\tilde{\alpha} \geqslant z_{i}^{+} ; \forall i \in\{1, \ldots, k\}$ then $(\tilde{x}, \tilde{\alpha})$ is a 


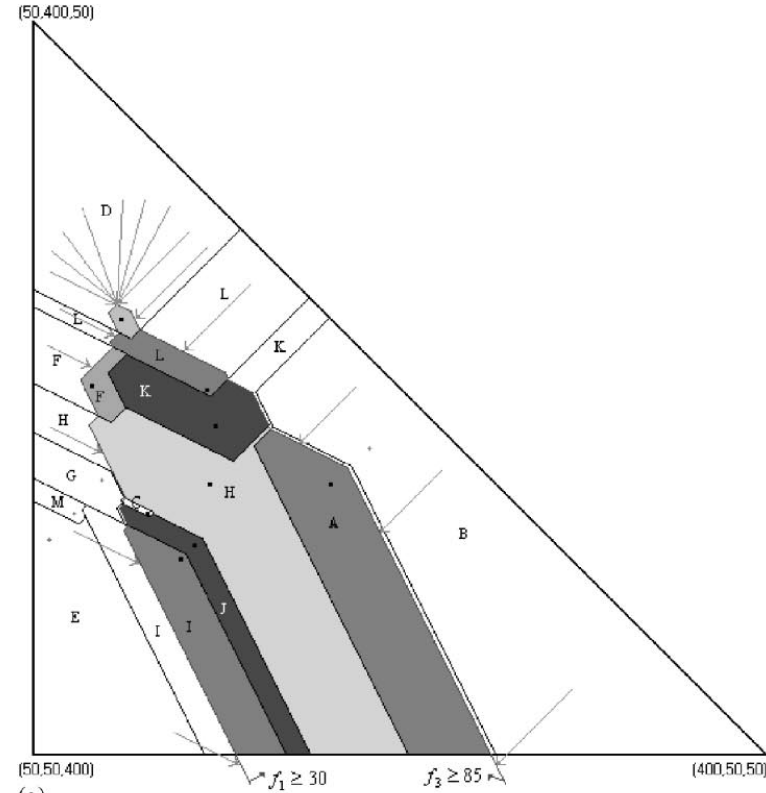

(a)

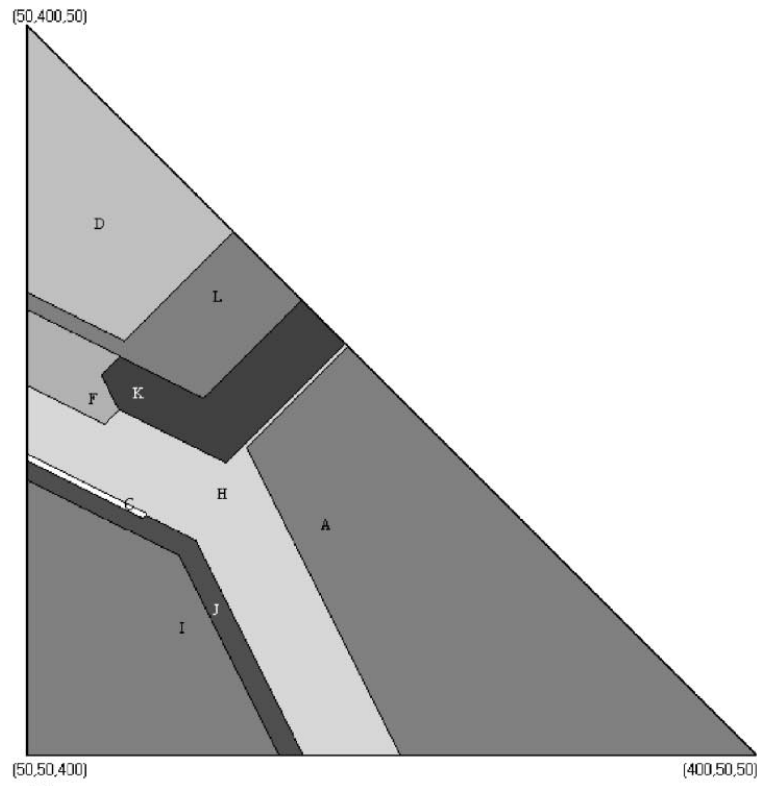

(b)

Figure 8. Indifference regions considering criterion bounds in $f_{1}$ and $f_{3}$ : reference point space of (a) the original problem and (b) the restricted problem.

feasible solution of $P_{1}\left(z^{++}\right)$. Let us suppose that it does not optimize $P_{1}\left(z^{++}\right)$and let $(\hat{x}, \hat{\alpha})$ be its optimal solution. Hence $\hat{\alpha}<\tilde{\alpha}$.

(2) Also,

$$
\begin{gathered}
\left\{\begin{array}{lc}
c^{j} \hat{x}+\hat{\alpha} \geqslant \max \left\{z_{j}^{+}, L_{j}+\tilde{\alpha}\right\} & j \in \bar{K} \\
c^{i} \hat{x}+\hat{\alpha} \geqslant z_{i}^{+} & i \notin \bar{K}
\end{array}\right. \\
\Leftrightarrow \begin{cases}c^{j} \hat{x}+\hat{\alpha} \geqslant L_{j}+\tilde{\alpha} & j \in \bar{K} \\
c^{i} \hat{x}+\hat{\alpha} \geqslant z_{i}^{+} & \forall i \in\{1, \ldots, k\}\end{cases}
\end{gathered}
$$

$(\hat{x}, \hat{\alpha})$ cannot be feasible for $P_{1}\left(z^{+}, L\right)$ because, from (1), it would be the optimal solution of $P_{1}\left(z^{+}, L\right)$. Therefore, there exists any $r \in \bar{K}$ such that $c^{r} \hat{x}<L_{r}$. But, from (2), $c^{r} \hat{x}+\hat{\alpha} \geqslant L_{r}+\tilde{\alpha} \Rightarrow$ $\tilde{\alpha}-\hat{\alpha} \leqslant c^{r} \hat{x}-L_{r}<0$. Hence $\tilde{\alpha}<\hat{\alpha}$ which contradicts (1) and thus the hypothesis that $(\hat{x}, \hat{\alpha})$ optimizes $P_{1}\left(z^{++}\right)$.

Note: Lemma 1 and Proposition 2 are still valid for $P_{2}$ provided that $\rho$ is positive small enough such that if $\bar{x}$ optimizes $P_{2}$ then $\bar{x}$ optimizes $P_{1}$. Hence, Proposition 2 can be used to translate indifference regions on the reference point space from the restricted problem to the original problem.

\section{Example}

Let us consider the tri-objective multiconstraint knapsack problem addressed in Section 3 and let us suppose that $f_{1} \geqslant 30$ and $f_{3} \geqslant 85$ were included to temporarily constrain the search for non-dominated solutions. Figure 8 (a) shows the indifference regions that would be defined from the reference point conversion established in Proposition 2. The non-dominated solutions ' $B$ ', 'E', ' $G$ ' and ' $M$ ' do not have representation because they do not satisfy the additional limitations. The reference points inside the shading areas remain unchanged by the mapping function. Reference points outside these areas are mapped onto the boundary following one of the directions $m_{1}$ or $m_{3}$ (except those that are mapped onto the upper vertex of the shading area). The arrows in Figure 8(a) illustrate it. The areas reserved to the other non-dominated solutions of the original problem are kept empty in this representation, but they could be filled if the additional limitations were eliminated. This graph can be compared with the graph of the Figure $8(\mathrm{~b})$ 
that shows the whole decomposition of the reference point space of the restricted problem. In Figure 8(b) the non-dominated solutions that satisfy the temporary constraints fill the triangle completely.

\section{CONCLUDING REMARKS}

In this paper, we have presented some properties of the reference points in Tchebycheff scalarizing functions for MOILP problems. We have analysed the shape of indifference regions on the reference point space, i.e. sets of reference points that lead to the same non-dominated solution. Although we could not establish a procedure for computing the whole indifference region of a non-dominated solution, we proposed a method of defining sub-regions in it. We have also exploited the decomposition of the reference point space when additional limitations on the objective function values are introduced.

MOILP problems with two or three objective functions enable the graphical representation of the indifference (sub)regions on the reference point space. Taking advantage of the directional searches for non-dominated solutions that we had implemented before, larger indifference sub-regions may be successively defined and appended. The graphical representation of these regions has been included in the computational system and our experience, namely with an application currently under study, have shown that this feature provides valuable information for the DM. It gives the DM new insights into the problem helping him/her to avoid selecting reference points that would lead to non-dominated solutions already known.

\section{REFERENCES}

Alves, MJ, Clímaco J. 1999. Using cutting planes in an interactive reference point approach for multiobjec- tive integer linear programming problems. European Journal of Operational Research 117(3): 565-577.

Alves MJ, Clímaco J. 2000. An interactive reference point approach for multiobjective mixedinteger programming using branch-and-bound. European Journal of Operational Research 124(3): 478-494.

Benayoun R, Montgolfier J, Tergny J, Larichev O. 1971. Linear programming with multiple objective functions: step method (STEM). Mathematical Programming 1: 366-375.

Durso A. 1992. An interactive combined branch-andbound/Tchebycheff algorithm for multiple criteria. In Multiple Criteria Decision Making, Goicoechea A Duckstein A, Zionts S (eds). Proceedings of the 9th Conference, Springer: Berlin; 107-122.

Jorge H, Antunes CH, Martins AG. 2000. A multiple objective decision support model for the selection of remote load control strategies. IEEE Transactions on Power Systems 5(2): 865-872.

Karaivanova J, Korhonen P, Narula S, Wallenius J, Vassilev V. 1995. A reference direction approach to multiple objective integer linear programming. European Journal of Operational Research 81: 176-187.

Korhonen P. Wallenius J. 1988. A Pareto race. Naval Research Logistics 35: 615-623.

Narula SC, Vassilev V. 1994. An interactive algorithm for solving multiple objective integer linear programming problems. European Journal of Operational Research 79: 443-450.

Steuer R, Choo E-U, 1983. An interactive weighted Tchebycheff procedure for multiple objective programming. Mathematical Programming 26: 326-344.

Vassilev V, Narula, SC. 1993. A reference direction algorithm for solving multiple objective integer linear programming problems. Journal of Operational Research Society 44(12): 1201-1209.

Wierzbicki A. 1980. The use of reference objectives in multiobjective optimization. In Multiple Criteria Decision Making, Theory and Application. Fandel G, Gal $\mathrm{T}$ (eds), Lecture Notes in Economics and Mathematical Systems, vol. 177. Springer: Berlin, 468-486.

Wierzbicki AP. 1998. Reference points in vector optimization and decision support. Interim Report IR-98-017, IIASA, Laxenburg, Austria. 